\title{
Output Feedback Tracking Control for a Class of Switched Nonlinear Systems with Time-varying Delay
}

\author{
Bin Wang Jun-Yong Zhai Shu-Min Fei \\ Key Laboratory of Measurement and Control of CSE, Ministry of Education, School of Automation, Southeast University, \\ Nanjing, Jiangsu 210096, China
}

\begin{abstract}
This paper studies the problem of tracking control for a class of switched nonlinear systems with time-varying delay. Based on the average dwell-time and piecewise Lyapunov functional methods, a new exponential stability criterion is obtained for the switched nonlinear systems. The designed output feedback $H_{\infty}$ controller can be obtained by solving a set of linear matrix inequalities (LMIs). Moreover, the proposed method does not need that a common Lyapunov function exists for the switched systems, and the switching signal just depends on time. A simulation example is provided to demonstrate the effectiveness of the proposed design scheme.
\end{abstract}

Keywords: Tracking control, switched nonlinear systems, exponential stability, time-varying delay, $H_{\infty}$ controller.

\section{Introduction}

Switched systems are a class of hybrid dynamical systems which consist of a family of subsystems and a rule that orchestrates the switching among them. The local behavior is affected by the continuous dynamical subsystems and the discrete dynamical switching mechanisms determine the global performance. In recent years, there are lots of significant achievements both in theory development and practical applications of switched systems ${ }^{[1-5]}$. On the other hand, time-delay systems are an important class of systems, which are ubiquitous in real world, such as in chemical process, aerodynamics, and communication networks systems. Sometimes, even a small delay may affect the system performance greatly. A stable system may become unstable or chaotic behavior may appear if delay is present in the system $^{[6-8]}$. Since the switched systems with time-delay have strong engineering background, they have attracted considerable attention, and some useful results have been obtained $^{[9-18]}$. There are several methods used in analyzing stability of time-delay switched systems, such as the common Lyapunov function, multiple Lyapunov functions, piecewise Lyapunov function and average dwell-time. The work in [12] gives delay-dependent conditions for the exponential stability of the switched linear systems with timevarying delay by common Lyapunov function. The work in [17] gives the exponential stability criterion for a class of switched linear systems with constant time delay by combining dwell-time with the piecewise Lyapunov function.

Tracking control for time-delay switched system is widely used in robot control and guided missile control. To the best of the authors' knowledge, the issue of tracking control has not been fully investigated for time-delay switched systems.

\footnotetext{
Regular paper

Special Issue on Recent Advances on Complex Systems Control, Modelling and Prediction II

Manuscript received September 12, 2013; accepted December 30, 2013

This work was supported by National Natural Science Foundation of China (Nos.61473082, 61273119, and 61104068), Six Talents Peaks Program of Jiangsu Province (No. 2014-DZXX-003) and the Fundamental Research Funds for the Central Universities (No. 2242013R30006).
}

Only a few results have been reported on tracking control for switched systems ${ }^{[19-26]}$. The stability of tracking control based on observer for time-delay switched linear systems has been investigated in [21], but the proposed method needs that a common Lyapunov function must exist for the switched systems. By using average dwell-time, a new exponential stability criterion of state feedback tracking control for switched nonlinear systems with time-varying delay has been obtained in [22]. In this paper, we aim to design an output feedback controller for a class of switched nonlinear systems with time-varying delay. Combining average dwell-time with the piecewise Lyapunov function, a new exponential stability criterion for a class of switched nonlinear systems with time-varying delay is derived. Moreover, when there exists a common Lyapunov function for the system, it will be exponentially stable under arbitrary switching law.

Notations. $\mathbf{R}^{n}$ denotes $n$-dimensional Euclidean space; $\mathbf{R}^{m \times n}$ denotes the space of $m \times n$ matrices with real entries. $L_{2}[0, \infty)$ is the space of square integrable functions on $[0, \infty)$, and $\mathscr{L}_{1}^{\text {loc }}\left([\varrho, \infty), \mathbf{R}^{n}\right)$ is the space of locally Lebesgue integrable vector valued functions on $[\varrho, \infty)$, where $\varrho$ is a scalar. For any given $\tau>0$, let $C_{n}=$ $C\left([-\tau, 0], \mathbf{R}^{n}\right)$ be the Banach space of continuous mapping from $\left([-\tau, 0], \mathbf{R}^{n}\right)$ to $\mathbf{R}^{n}$ with the topology of uniform convergence. $I$ represents identity matrix with appropriate dimension. $P>0(\geqslant,<, \leqslant 0)$ denotes a positive definite (positive semi-defined, negative definite, negative semi-definite) matrix. $\lambda_{\min }(\cdot)$ and $\lambda_{\max }(\cdot)$ denote the minimal and maximal eigenvalues of a square matrix. $\sigma_{\max }(\cdot)$ means the maximal singular value of a matrix. The superscript "T" stands for matrix transpose and the symmetric terms in a symmetric matrix are denoted by $*$. Let $x_{t} \in C_{n}$ be defined by $x_{t}(\theta)=x(t+\theta), \theta \in[-\tau, 0] .\|\cdot\|$ denotes the usual 2norm, and $\left\|x_{t}\right\|_{c l}=\sup _{-\tau \leqslant \theta \leqslant 0}\{\|x(t+\theta)\|,\|\dot{x}(t+\theta)\|\}$. 


\section{Problem formulation and preliminar- ies}

Consider the switched nonlinear system with timevarying delay

$$
\begin{aligned}
& \dot{x}(t)=f_{\sigma}(x(t))+A_{\sigma} x(t)+D_{\sigma} x(t-d(t))+B_{\sigma} u(t)+\omega(t) \\
& y(t)=C_{\sigma} x(t) \\
& x(t)=\varphi(t), \quad-\tau \leqslant t \leqslant 0
\end{aligned}
$$

where $x \in \mathbf{R}^{n}$ is the system state vector, $u(t) \in \mathbf{R}^{p}$ is the control input, $y(t) \in \mathbf{R}^{q}$ is the output, $\omega(t) \in \mathbf{R}^{n}$ is the bounded exogenous disturbance which belongs to $L_{2}[0, \infty)$ and $\mathscr{L}_{1}^{\text {loc }}\left([\varrho, \infty), \mathbf{R}^{n}\right)$. $f_{\sigma}(\cdot): \mathbf{R}^{n} \rightarrow \mathbf{R}^{n}$ is a known nonlinear function; $\sigma(t):\left[t_{0}, \infty\right) \rightarrow M \triangleq\left\{1,2, \cdots, m_{0}\right\}$ is the switching signal. $d(t)$ denotes the time-varying delay satisfying $0<d(t) \leqslant \tau ; \varphi(t)$ is a continuous vector-valued initial function. Moreover, $\sigma(t)=i$ means that the $i$-th subsystem is activated, $A_{i}, B_{i}, C_{i}, D_{i}$ are known constant matrices with appropriate dimensions. Without loss of generality, we state that the following assumptions hold.

Assumption 1. $U_{i} \in \mathbf{R}^{n \times n}, i \in M$ are known constant matrices, the function $f_{i}(x(t))$ is Lipschitz for all $x(t) \in \mathbf{R}^{n}$ and $\hat{x}(t) \in \mathbf{R}^{n}$, and satisfies

$$
\left\|f_{i}(x(t))-f_{i}(\hat{x}(t))\right\| \leqslant\left\|U_{i}(x(t)-\hat{x}(t))\right\| .
$$

Assumption 2. For $i \in M$, the subsystem $\left(A_{i}, C_{i}, D_{i}\right)$ are detectable ${ }^{[21]}$ and $B_{i}$ are full row rank.

Definition $\mathbf{1}^{[10]}$. System (1) is said to be exponentially stabilizable under control law $u(t)$ and switching law $\sigma(t)$, if the solution $x(t)$ of system (1) through $\left(t_{0}, \varphi\right) \in \mathbf{R}_{+} \times C_{n}$ satisfies

$$
\|x(t)\| \leqslant \kappa\left\|x_{t_{0}}\right\|_{c l} \mathrm{e}^{-\lambda\left(t-t_{0}\right)}, \quad \forall t \geqslant t_{0}
$$

for some constants $\kappa \geqslant 0$ and $\lambda>0$.

Suppose that the state observer is of the form

$$
\begin{aligned}
\dot{\hat{x}}(t)= & f_{\sigma}(\hat{x}(t))+A_{\sigma} \hat{x}(t)+D_{\sigma} \hat{x}(t-d(t))+ \\
& B_{\sigma} u(t)+L_{\sigma}(y(t)-\hat{y}(t)) \\
\hat{y}(t)= & C_{\sigma} \hat{x}(t)
\end{aligned}
$$

where $y(t)$ is the measurable output of system (1), and $L_{\sigma}$ is the observer gain matrix to be determined later.

The reference model is given as

$$
\dot{x}_{r}(t)=A_{r} x_{r}(t)+r(t)
$$

where $x_{r}(t) \in \mathbf{R}^{n}$ is the reference state, $A_{r}$ is a Hurwitz matrix, $r(t)$ is the bounded reference input which belongs to $L_{2}[0, \infty)$ and $\mathscr{L}_{1}^{\text {loc }}\left([\varrho, \infty), \mathbf{R}^{n}\right)$, respectively.

Now, define tracking error $e_{r}(t)=x(t)-x_{r}(t)$, and consider the $H_{\infty}$ tracking performance as ${ }^{[22]}$

$$
\int_{t_{0}}^{\infty} \mathrm{e}^{-\alpha\left(t-t_{0}\right)} e_{r}^{\mathrm{T}}(t) e_{r}(t) \mathrm{d} t \leqslant \gamma^{2} \int_{t_{0}}^{\infty} \bar{\omega}^{\mathrm{T}}(t) \bar{\omega}(t) \mathrm{d} t, t \geqslant t_{0}
$$

where $\bar{\omega}(t)=\left[\omega^{\mathrm{T}}(t), r^{\mathrm{T}}(t)\right]^{\mathrm{T}}, \alpha, \gamma$ are positive constants.

Define the difference between the real state and the observer state, the observer state and the reference state as

$$
e(t)=x(t)-\hat{x}(t), \quad \hat{e}_{r}(t)=\hat{x}(t)-x_{r}(t) .
$$

Design the output feedback controller

$$
u(t)=K_{\sigma} \hat{x}(t)+F_{\sigma} x_{r}(t)-B_{\sigma}^{\mathrm{T}}\left(B_{\sigma} B_{\sigma}^{\mathrm{T}}\right)^{-1} f_{\sigma}(\hat{x}(t))
$$

where $K_{\sigma}, F_{\sigma}$ are the output feedback gains. Combining $(1),(4),(5)$ and $(7)$, we can obtain the augmented systems

$$
\begin{aligned}
& \dot{e}(t)=\left(A_{\sigma}-L_{\sigma} C_{\sigma}\right) e(t)+D_{\sigma} e(t-d(t))+f_{\sigma}(x(t))- \\
& f_{\sigma}(\hat{x}(t))+\omega(t) \\
& \dot{\hat{x}}(t)=\left(A_{\sigma}+B_{\sigma} K_{\sigma}\right) \hat{x}(t)+B_{\sigma} F_{\sigma} x_{r}(t)+D_{\sigma} \hat{x}(t-d(t))+ \\
& L_{\sigma} C_{\sigma} e(t) \\
& \dot{x}_{r}(t)=A_{r} x_{r}(t)+r(t) \text {. } \\
& \bar{x}(t)=\left[\begin{array}{c}
\hat{x}(t) \\
x_{r}(t)
\end{array}\right], \quad \bar{A}_{\sigma}=\left[\begin{array}{cc}
A_{\sigma}+B_{\sigma} K_{\sigma} & B_{\sigma} F_{\sigma} \\
0 & A_{r}
\end{array}\right], \\
& g_{\sigma}(t)=\left[\begin{array}{c}
L_{\sigma} C_{\sigma} e(t) \\
r(t)
\end{array}\right], \quad \bar{D}_{\sigma}=\left[\begin{array}{cc}
D_{\sigma} & 0 \\
0 & 0
\end{array}\right] .
\end{aligned}
$$

Then, system (9) can be rewritten as

$$
\dot{\bar{x}}(t)=\bar{A}_{\sigma} \bar{x}(t)+\bar{D}_{\sigma} \bar{x}(t-d(t))+g_{\sigma}(t) .
$$

Define the switching sequences of system (8) and (9)

$$
\Sigma \triangleq\left\{\left(i_{0}, t_{0}\right),\left(i_{1}, t_{1}\right), \cdots,\left(i_{k}, t_{k}\right), \cdots \mid i_{k} \in M\right\}
$$

which means the $i_{k}$-th subsystem is activated at time $t_{k}$.

Definition $2^{[20,21]}$. For system (1), if there exist control input $u(t)$ and switching law $\sigma(t)$, such that: 1) the closedloop (8) and (9) are exponentially stable when $\bar{\omega}(t) \equiv 0 ; 2)$ performance index $(6)$ is satisfied when $\bar{\omega}(t) \neq 0$ under zero initial conditions, that is, $x(t)=0, x_{r}(0)=0, \hat{x}(t)=0, t \in$ $[-\tau, 0]$. Then system (1) is said to have observer-based $H_{\infty}$ model reference tracking performance.

Definition $3^{[1]}$. For the switched signal $\sigma(t)$ and any $t \geqslant \tau \geqslant 0, N_{\sigma}(t, \tau)$ denotes the system switching times in the open interval $(\tau, t)$. If

$$
N_{\sigma}(t, \tau) \leqslant N_{0}+\frac{t-\tau}{\tau_{a}}
$$

holds for $\tau_{a}>0$ and $N_{0} \geqslant 0$, then $\tau_{a}$ is called average dwell-time. Without loss of generality, as commonly used in the literature, we assume $N_{0}=0$.

To conclude this section, we recall the following lemmas.

Lemma $1^{[9]}$. Let $U, V$ be real matrices of appropriate dimensions. Then, for any matrix $Q>0$ of appropriate dimension and scalar $\epsilon>0$, it holds that

$$
U V+V^{\mathrm{T}} U^{\mathrm{T}} \leqslant \epsilon^{-1} U Q^{-1} U^{\mathrm{T}}+\epsilon V^{\mathrm{T}} Q V
$$

Lemma $\mathbf{2}^{[13]}$. For any constant matrix $N>0$, scalar $\tau>0$, any $t \in[0,+\infty)$, vector function $y:[t-\tau, t] \rightarrow \mathbf{R}^{n}$, such that the integrations in the following are well defined, then

$$
\left(\int_{t-\tau}^{t} y(s) \mathrm{d} s\right)^{\mathrm{T}} N \int_{t-\tau}^{t} y(s) \mathrm{d} s \leqslant \tau \int_{t-\tau}^{t} y(s)^{\mathrm{T}} N y(s) \mathrm{d} s .
$$

Consider the linear time-varying delay system (10) without switching and its homogeneous system as

$$
\begin{gathered}
\dot{\bar{x}}(t)=\bar{A} \bar{x}(t)+\bar{D} \bar{x}(t-d(t))+g(t) \\
\dot{\bar{x}}(t)=\bar{A} \bar{x}(t)+\bar{D} \bar{x}(t-d(t)) .
\end{gathered}
$$


It can be rewritten in operator form ${ }^{[21]}$

$$
\begin{aligned}
& \dot{\bar{x}}(t)=L\left(t, x_{t}\right)+g(t), \quad t \geqslant \varrho \\
& \dot{\bar{x}}(t)=L\left(t, x_{t}\right), \quad \bar{x}_{\varrho}=\phi, \quad t \geqslant \varrho
\end{aligned}
$$

where the operator $L(t, \phi)$ is linear in $\phi$, and has the form $L(t, \phi)=A \phi(0)+D \phi(-d(t))$, in which $\phi(\theta)=x(t+\theta), \theta \in$ $[-\tau, 0]$. Suppose there is an $m \in \mathscr{L}_{1}^{\text {loc }}\left([\varrho, \infty), \mathbf{R}_{+}\right)$such that

$$
|L(t, \phi)| \leqslant m(t)|\phi|
$$

for all $t \in(-\infty, \infty), \phi \in \mathbf{C}_{n}$

Lemma 3 (Variation-of-constants). Let $\bar{x}(\varrho, \phi, g)(t)$ denote the solution of system $(13)$, and $\bar{x}(\varrho, \phi, 0)(t)$ denote the solution of the corresponding homogeneous system (14). Denote $\bar{x}(\varrho, \phi, 0)(t+\theta)$ by $\bar{x}_{t}(\varrho, \phi, 0)(\theta),-\tau \leqslant \theta \leqslant 0$, $X_{0}(\theta)=\left\{\begin{array}{l}0, \quad-\tau \leqslant \theta<0 \\ I, \quad \theta=0\end{array}\right.$. If $\bar{x}_{t}(\varrho, \phi, 0) \triangleq T(t, \varrho) \phi$, then $T(t, \varrho)$ is a continuous linear operator. And if (15) is satisfied and $g(t) \in \mathscr{L}_{1}^{\text {loc }}\left([\varrho, \infty), \mathbf{R}^{2 n}\right)$, then

$$
\bar{x}_{t}(\varrho, \phi, g)=T(t, \varrho) \phi+\int_{\varrho}^{t} T(t, s) X_{0} g(s) \mathrm{d} s, \quad t \geqslant \varrho .
$$

Proof. The proof follows the same lines as in $[21,27]$.

\section{Main results}

\subsection{Observer gain matrix design}

Consider the simplified system of (8) when $\omega(t)=0$

$$
\begin{aligned}
\dot{e}(t)= & \left(A_{\sigma}-L_{\sigma} C_{\sigma}\right) e(t)+D_{\sigma} e(t-d(t))+ \\
& f_{\sigma}(x(t))-f_{\sigma}(\hat{x}(t)) .
\end{aligned}
$$

Theorem 1. For system (17), if there exist scalar $\alpha>$ $0, \tau>0$, matrices $H_{i}>0, G_{i}>0, \bar{L}_{i}, \forall i \in M$ and any invertible matrix $Y_{i}$ with appropriate dimensions, such that the following LMIs hold

$$
\Theta_{i}=\left[\begin{array}{cccc}
\varphi_{11 i} & \varphi_{12 i} & Y_{i} & -\tau Y_{i} D_{i} \\
* & \varphi_{22 i} & Y_{i} & -\tau Y_{i} D_{i} \\
* & * & -I & 0 \\
* & * & * & -\tau \mathrm{e}^{-\alpha \tau} G_{i}
\end{array}\right]<0
$$

and the average dwell-time satisfies

$$
\tau_{a_{1}}>\frac{\ln \mu_{1}}{\alpha}
$$

where $\varphi_{11 i}=Y_{i}\left(A_{i}+D_{i}\right)+\left(A_{i}+D_{i}\right)^{\mathrm{T}} Y_{i}^{\mathrm{T}}-\bar{L}_{i} C_{i}-C_{i}^{\mathrm{T}} \bar{L}_{i}^{\mathrm{T}}+$ $\alpha H_{i}+U_{i}^{\mathrm{T}} U_{i}, \varphi_{12 i}=H_{i}-Y_{i}+\left(A_{i}+D_{i}\right)^{\mathrm{T}} Y_{i}^{\mathrm{T}}-C_{i}^{\mathrm{T}} \bar{L}_{i}^{\mathrm{T}}, \varphi_{22 i}=$ $\tau G_{i}-Y_{i}-Y_{i}^{\mathrm{T}}$ and $\mu_{1} \geqslant 1$ satisfies

$$
H_{i} \leqslant \mu_{1} H_{j}, \quad G_{i} \leqslant \mu_{1} G_{j}, \quad \forall i, j \in M, i \neq j .
$$

Then, system (17) is exponentially stable and the observer gain matrix is given by $L_{i}=Y_{i}^{-1} \bar{L}_{i}$.

Proof. Choose the piecewise Lyapunov functional candidate as

$$
\begin{aligned}
& V(e(t))=V_{\sigma}(e(t))=V_{1 \sigma}(e(t))+V_{2 \sigma}(e(t)) \\
& V_{1 \sigma}(e(t))=e^{\mathrm{T}}(t) H_{\sigma} e(t) \\
& V_{2 \sigma}(e(t))=\int_{-\tau}^{0} \int_{t+\theta}^{t} \mathrm{e}^{-\alpha(t-s)} \dot{e}^{\mathrm{T}}(s) G_{\sigma} \dot{e}(s) \mathrm{d} s \mathrm{~d} \theta .
\end{aligned}
$$

During any interval $\left[t_{k}, t_{k+1}\right)$, we let $\sigma(t)=i_{k}=i$, then $V(e(t))=V_{i}(e(t))$. Along the trajectories of system (17), the time derivative of $V_{i}(e(t))$ is given as

$$
\begin{aligned}
\dot{V}_{i}(e(t))= & 2 e^{\mathrm{T}}(t) H_{i} \dot{e}(t)-\alpha V_{2 i}(e(t))+\tau \dot{e}^{\mathrm{T}}(t) G_{i} \dot{e}(t)- \\
& \int_{t-\tau}^{t} \mathrm{e}^{-\alpha(t-s)} \dot{e}^{\mathrm{T}}(s) G_{i} \dot{e}(s) \mathrm{d} s .
\end{aligned}
$$

From Assumption 1, we can get

$$
\begin{aligned}
& {\left[f_{i}(x(t))-f_{i}(\hat{x}(t))\right]^{\mathrm{T}}\left[f_{i}(x(t))-f_{i}(\hat{x}(t))\right] \leqslant} \\
& \quad e^{\mathrm{T}}(t) U_{i}^{\mathrm{T}} U_{i} e(t) .
\end{aligned}
$$

Substituting (23) into (22), one has

$$
\begin{aligned}
& \dot{V}_{i}(e(t))+\alpha V_{i}(e(t)) \leqslant e^{\mathrm{T}}(t)\left(\alpha H_{i}+U_{i}^{\mathrm{T}} U_{i}\right) e(t)+ \\
& 2 e^{\mathrm{T}}(t) H_{i} \dot{e}(t)+\tau \dot{e}^{\mathrm{T}}(t) G_{i} \dot{e}(t)-\mathrm{e}^{-\alpha \tau} \int_{t-d(t)}^{t} \dot{e}^{\mathrm{T}}(s) G_{i} \dot{e}(s) \mathrm{d} s- \\
& {\left[f_{i}(x(t))-f_{i}(\hat{x}(t))\right]^{\mathrm{T}}\left[f_{i}(x(t))-f_{i}(\hat{x}(t))\right] .}
\end{aligned}
$$

For the free weighting matrix $Y_{i}$ and $\int_{t-d(t)}^{t} \dot{e}(s) \mathrm{d} s=e(t)-$ $e(t-d(t))$, it holds that

$$
\begin{gathered}
2\left[e^{\mathrm{T}}(t), \dot{e}^{\mathrm{T}}(t)\right]\left[\begin{array}{c}
Y_{i} \\
Y_{i}
\end{array}\right]\left[\left(A_{i}+D_{i}-L_{i} C_{i}\right) e(t)-\dot{e}(t)-\right. \\
\left.D_{i} \int_{t-d(t)}^{t} \dot{e}(s) \mathrm{d} s+f_{i}(x(t))-f_{i}(\hat{x}(t))\right]=0 .
\end{gathered}
$$

Let $\xi(t, s)=\left[e^{\mathrm{T}}(t), \dot{e}^{\mathrm{T}}(t), f_{i}^{\mathrm{T}}(x(t))-f_{i}^{\mathrm{T}}(\hat{x}(t)), \dot{e}^{\mathrm{T}}(s)\right]^{\mathrm{T}}$. Substituting (25) into (24), it yields

$$
\begin{gathered}
\dot{V}_{i}(e(t))+\alpha V_{i}(e(t)) \leqslant \frac{1}{d(t)} \int_{t-d(t)}^{t} \xi^{\mathrm{T}}(t, s) \bar{\Theta}_{i} \xi(t, s) \mathrm{d} s \quad(26) \\
\text { where } \bar{\Theta}_{i}=\left[\begin{array}{cccc}
\varphi_{11 i} & \varphi_{12 i} & Y_{i} & -d(t) Y_{i} D_{i} \\
* & \varphi_{22 i} & Y_{i} & -d(t) Y_{i} D_{i} \\
* & * & -I & 0 \\
* & * & * & -d(t) \mathrm{e}^{-\alpha \tau} G_{i}
\end{array}\right]<0 . \quad \text { By }
\end{gathered}
$$
proper transformation to (18), we can get $\bar{\Theta}_{i}<0$, then

$$
\dot{V}_{i}(e(t))+\alpha V_{i}(e(t)) \leqslant 0 .
$$

Let $t_{k}^{-}$be the left limit of $t_{k}$, which is before the switching at $t_{k}$. Using (20) and (21), one has

$$
V_{i}\left(e\left(t_{k}\right)\right) \leqslant \mu_{1} V_{j}\left(e\left(t_{k}^{-}\right)\right), \quad \forall i, j \in M, \quad i \neq j .
$$

Let any $t \in\left[t_{k}, t_{k+1}\right)$, then $N_{\sigma}\left(t, t_{0}\right)=k$. Combining (11), (27) and (28), we can obtain

$$
\begin{aligned}
V_{i_{k}}(e(t)) \leqslant & V_{i_{k}}\left(e\left(t_{k}\right)\right) \mathrm{e}^{-\alpha\left(t-t_{k}\right)} \leqslant \\
& \mu_{1} V_{i_{k-1}}\left(e\left(t_{k-1}\right)\right) \mathrm{e}^{-\alpha\left(t-t_{k-1}\right)} \leqslant \cdots \leqslant \\
& \mu_{1}^{k} V_{i_{0}}\left(e\left(t_{0}\right)\right) \mathrm{e}^{-\alpha\left(t-t_{0}\right)}= \\
& \mathrm{e}^{-\alpha\left(t-t_{0}\right)+k \ln \mu_{1}} V_{i_{0}}\left(e\left(t_{0}\right)\right) .
\end{aligned}
$$

From Definition 3, we can get $k \ln \mu_{1} \leqslant \frac{t-t_{0}}{\tau_{a_{1}}} \ln \mu_{1}$. Let $2 \lambda=\alpha-\frac{\ln \mu_{1}}{\tau_{a_{1}}}>0$, then,

$$
a\|e(t)\|^{2} \leqslant \mathrm{e}^{-2 \lambda\left(t-t_{0}\right)} V_{i_{0}}\left(e\left(t_{0}\right)\right) \leqslant b \mathrm{e}^{-2 \lambda\left(t-t_{0}\right)}\left\|e_{t_{0}}\right\|_{c l}^{2}
$$


where $a=\min _{i \in M}\left\{\lambda_{\min }\left(H_{i}\right)\right\}, b=\max _{i \in M}\left\{\lambda_{\max }\left(H_{i}\right)+\right.$ $\left.\frac{\tau^{2}}{2} \lambda_{\max }\left(G_{i}\right)\right\}$. From (30), we can obtain

$$
\|e(t)\| \leqslant \sqrt{\frac{b}{a}} \mathrm{e}^{-\lambda\left(t-t_{0}\right)}\left\|e_{t_{0}}\right\|_{c l} .
$$

By Definition 1, we know that system (17) is exponentially stable.

\subsection{Stability and $H_{\infty}$ performance}

Theorem 2. For system (10), if there exist scalars $\alpha>$ $0, \tau>0, \gamma>0$, and matrices $P_{i}>0, S_{i}>0, \forall i \in M$, such that the following inequalities hold

$$
\Phi_{i}=\left[\begin{array}{cccc}
\phi_{11 i} & P_{i} \bar{D}_{i} & P_{i} & \tau\left(\bar{A}_{i}+\bar{D}_{i}\right)^{\mathrm{T}} \\
* & \phi_{22 i} & 0 & \tau \bar{D}_{i}^{\mathrm{T}} \\
* & * & -\frac{1}{2} \gamma^{2} I & \tau I \\
* & * & * & -S_{i}^{-1}
\end{array}\right]<0
$$

and the average dwell-time satisfies

$$
\tau_{a}=\max \left\{\tau_{a_{1}}, \tau_{a_{2}}\right\}, \quad \tau_{a_{2}}>\frac{\ln \mu_{2}}{\alpha}
$$

where $\phi_{11 i}=P_{i}\left(\bar{A}_{i}+\bar{D}_{i}\right)+\left(\bar{A}_{i}+\bar{D}_{i}\right)^{\mathrm{T}} P_{i}+\alpha P_{i}+\bar{Q}, \phi_{22 i}=$ $-\mathrm{e}^{-\alpha \tau} S_{i}, \bar{Q}=\left[\begin{array}{cc}I & -I \\ -I & I\end{array}\right]$, and $\mu_{2} \geqslant 1$ satisfies

$$
P_{i} \leqslant \mu_{2} P_{j}, \quad S_{i} \leqslant \mu_{2} S_{j}, \quad \forall i, j \in M, i \neq j .
$$

Then system (10) is exponentially stable, and the $H_{\infty}$ model reference tracking performance in (1) is guaranteed.

Proof. From (32), using Schur complement, it yields

$$
\begin{aligned}
& \Lambda_{i}=\left[\begin{array}{ccc}
\phi_{11 i}^{\prime} & P_{i} \bar{D}_{i} & P_{i} \\
* & \phi_{22 i} & 0 \\
* & * & -\frac{1}{2} \gamma^{2} I
\end{array}\right]+\tau^{2}\left[\bar{A}_{i}+\bar{D}_{i} \quad \bar{D}_{i} \quad I\right]^{\mathrm{T}} \\
& S_{i}\left[\bar{A}_{i}+\bar{D}_{i} \quad \bar{D}_{i} \quad I\right]<\left[\begin{array}{ccc}
-\bar{Q} & 0 & 0 \\
0 & 0 & 0 \\
0 & 0 & 0
\end{array}\right]
\end{aligned}
$$

where $\phi_{11 i}^{\prime}=\phi_{11 i}-\bar{Q}$.

First, consider the nominal system of system (10):

$$
\dot{\bar{x}}(t)=\bar{A}_{\sigma} \bar{x}(t)+\bar{D}_{\sigma} \bar{x}(t-d(t))
$$

Define the piecewise Lyapunov functional candidate as

$$
\begin{aligned}
& V(\bar{x}(t))=V_{\sigma}(\bar{x}(t))=V_{1 \sigma}(\bar{x}(t))+V_{2 \sigma}(\bar{x}(t)) \\
& V_{1 \sigma}(\bar{x}(t))=\bar{x}^{\mathrm{T}}(t) P_{\sigma} \bar{x}(t) \\
& V_{2 \sigma}(\bar{x}(t))=\tau \int_{-\tau}^{0} \int_{t+\theta}^{t} \mathrm{e}^{-\alpha(t-s)} \dot{\bar{x}}^{\mathrm{T}}(s) S_{\sigma} \dot{\bar{x}}(s) \mathrm{d} s \mathrm{~d} \theta .
\end{aligned}
$$

Let $-\int_{t-d(t)}^{t} \dot{\bar{x}}(s) \mathrm{d} s=z(t)=\bar{x}(t-d(t))-\bar{x}(t)$, then $\dot{\bar{x}}(t)=$ $\left(\bar{A}_{i}+\bar{D}_{i}\right) \bar{x}(t)+\bar{D}_{i} z(t)$. Then, along the trajectories of system (36), the time derivative of $V_{i}(\bar{x}(t))$ is given by

$$
\begin{aligned}
\dot{V}_{i}(\bar{x}(t)) \leqslant & \bar{x}^{\mathrm{T}}(t)\left[P_{i}\left(\bar{A}_{i}+\bar{D}_{i}\right)+\left(\bar{A}_{i}+\bar{D}_{i}\right)^{\mathrm{T}} P_{i}\right] \bar{x}(t)+ \\
& 2 \bar{x}^{\mathrm{T}}(t) P_{i} \bar{D}_{i} z(t)-\alpha V_{2 i}(\bar{x}(t))+\tau^{2} \dot{\bar{x}}^{\mathrm{T}}(t) S_{i} \dot{\bar{x}}(t)- \\
& \tau \mathrm{e}^{-\alpha \tau} \int_{t-d(t)}^{t} \dot{\bar{x}}^{\mathrm{T}}(s) S_{i} \dot{\bar{x}}(s) \mathrm{d} s
\end{aligned}
$$

By Lemma 2, it can be obtained that

$$
-\tau \int_{t-d(t)}^{t} \dot{\bar{x}}^{\mathrm{T}}(s) S_{i} \dot{\bar{x}}(s) \mathrm{d} s \leqslant-z^{\mathrm{T}}(t) S_{i} z(t) .
$$

Let $\eta(t)=\left[\bar{x}^{\mathrm{T}}(t), z^{\mathrm{T}}(t)\right]^{\mathrm{T}}$. Substituting (38) into (37), it yields

$$
\dot{V}_{i}(\bar{x}(t))+\alpha V_{i}(\bar{x}(t)) \leqslant \eta^{\mathrm{T}}(t) \bar{\Phi}_{i} \eta(t)
$$

where

$$
\begin{aligned}
& \bar{\Phi}_{i}=\left[\begin{array}{cc}
P_{i}\left(\bar{A}_{i}+\bar{D}_{i}\right)+\left(\bar{A}_{i}+\bar{D}_{i}\right)^{\mathrm{T}} P_{i}+\alpha P_{i} & P_{i} \bar{D}_{i} \\
* & -\mathrm{e}^{-\alpha \tau} S_{i}
\end{array}\right]+ \\
& \tau^{2}\left[\begin{array}{ll}
\bar{A}_{i}+\bar{D}_{i} & \bar{D}_{i}
\end{array}\right]^{\mathrm{T}} S_{i}\left[\begin{array}{cc}
\bar{A}_{i}+\bar{D}_{i} & \bar{D}_{i}
\end{array}\right]
\end{aligned}
$$

From (35), (39) and (40), one can obtain

$$
\dot{V}_{i}(\bar{x}(t))+\alpha V_{i}(\bar{x}(t)) \leqslant 0 .
$$

By using the same means as the proof of Theorem 1, we can get

$$
\|\bar{x}(t)\| \leqslant \sqrt{\frac{b_{1}}{a_{1}}} \quad \mathrm{e}^{-\lambda_{1}\left(t-t_{0}\right)}\left\|\bar{x}_{t_{0}}\right\|_{c l}
$$

where $2 \lambda_{1}=\alpha-\frac{\ln \mu_{2}}{\tau_{a_{2}}}>0, a_{1}=\min _{i \in M}\left\{\lambda_{\min }\left(P_{i}\right)\right\}, b_{1}=$ $\max _{i \in M}\left\{\lambda_{\max }\left(P_{i}\right)+\frac{\tau^{3}}{2} \lambda_{\max }\left(S_{i}\right)\right\}$. Then, system (36) is exponentially stable.

Next, the stability of system (10) will be analysed with $g_{i}(t) \neq 0$ when $\bar{\omega}(t)=0, g_{i}(t)=\left[\begin{array}{c}L_{i} C_{i} e(t) \\ 0\end{array}\right]$. Since $g_{\sigma}(t) \in$ $\mathscr{L}_{1}^{\text {loc }}\left([\varrho, \infty), \mathbf{R}^{2 n}\right)$, for any $t \in\left[t_{j}, t_{j+1}\right)$, by Lemma 3 , we get the solution of (10) with the initial condition $\left(t_{0}, \phi_{i_{0}}\right)$ :

$$
\begin{gathered}
\bar{x}_{t}\left(t_{0}, \phi_{i_{0}}, g_{\sigma}\right)=T_{i_{j}}\left(t, t_{j}\right) \phi_{i_{j}}+\int_{t_{j}}^{t} T(t, s) X_{0} g_{i_{j}}(s) \mathrm{d} s= \\
T_{i_{j}}\left(t, t_{j}\right) T_{i_{j-1}}\left(t_{j}, t_{j-1}\right) \phi_{i_{j-1}}+T_{i_{j}}\left(t, t_{j}\right) \\
\int_{t_{j-1}}^{t_{j}} T\left(t_{j}, s\right) X_{0} g_{i_{j-1}}(s) \mathrm{d} s+\int_{t_{j}}^{t} T(t, s) X_{0} g_{i_{j}}(s) \mathrm{d} s=\cdots= \\
T\left(t, t_{0}\right) \phi_{i_{0}}+\int_{t_{0}}^{t} T(t, s) X_{0} g_{\sigma}(s) \mathrm{d} s
\end{gathered}
$$

where $T\left(t, t_{0}\right)=T_{i_{j}}\left(t, t_{j}\right) \cdot T_{i_{j-1}}\left(t_{j}, t_{j-1}\right) \cdots T_{i_{0}}\left(t_{1}, t_{0}\right)$ is a continuous piecewise linear operator.

Recalling the above analysis, system (36) is exponentially stable. There exist $\eta_{1}>0, \kappa_{1}>0$ such that

$$
\begin{gathered}
\left\|T\left(t, t_{0}\right)\right\| \leqslant \kappa_{1} \mathrm{e}^{-\eta_{1}\left(t-t_{0}\right)} \\
\left\|T(t, s) X_{0}\right\| \leqslant \kappa_{1} \mathrm{e}^{-\eta_{1}(t-s)}, \quad t \geqslant s \geqslant t_{0} .
\end{gathered}
$$

If (18), (19) and (20) hold, system (8) is exponentially stable by Theorem 1. From (31), there exists a scalar $B_{0}=\sqrt{\frac{b}{a}}$ $\left\|e_{t_{0}}\right\|_{c l} \max _{i \in M}\left\{\sigma_{\max }\left(L_{i} C_{i}\right)\right\}$, such that

$$
\left\|g_{\sigma}(t)\right\| \leqslant\left\|L_{\sigma} C_{\sigma}\right\|\|e(t)\| \leqslant B_{0} \mathrm{e}^{-\lambda\left(t-t_{0}\right)}, \quad t \geqslant t_{0} .
$$

Substituting (44) and (45) into (43), and choosing $\eta_{1} \neq \lambda$, 
it yields

$$
\begin{aligned}
& \left\|\bar{x}_{t}\left(t_{0}, \phi_{i_{0}}, g_{\sigma}\right)\right\| \leqslant \kappa_{1} \mathrm{e}^{-\eta_{1}\left(t-t_{0}\right)}\left\|\phi_{i_{0}}\right\|+ \\
& \int_{t_{0}}^{t} \kappa_{1} \mathrm{e}^{-\eta_{1}(t-s)} B_{0} \mathrm{e}^{-\lambda\left(s-t_{0}\right)} \mathrm{d} s \leqslant \\
& \kappa_{1} \mathrm{e}^{-\eta_{1}\left(t-t_{0}\right)}\left\|\phi_{i_{0}}\right\|+\frac{\kappa_{1} B_{0}}{\eta_{1}-\lambda}\left(\mathrm{e}^{-\lambda\left(t-t_{0}\right)}-\mathrm{e}^{-\eta_{1}\left(t-t_{0}\right)}\right) \leqslant \\
& \kappa_{1}\left(\left\|\phi_{i_{0}}\right\|+\frac{B_{0}}{\left|\eta_{1}-\lambda\right|}\right) \mathrm{e}^{-\beta\left(t-t_{0}\right)}
\end{aligned}
$$

where $\beta=\min \left\{\lambda, \eta_{1}\right\}$. We know that system (10) is exponentially stable when $\bar{\omega}(t)=0$.

Second, we will prove that performance index (6) is satisfied under zero initial condition $\bar{x}\left(t_{0}\right)=0, e\left(t_{0}\right)=0$ with $\bar{\omega}(t) \neq 0$. Let $\varsigma(t)=\left[\bar{x}^{\mathrm{T}}(t), z^{\mathrm{T}}(t), g_{i}^{\mathrm{T}}(t)\right]^{\mathrm{T}}$. Using the same method as the above analysis, it implies

$$
\begin{aligned}
& \dot{V}_{i}(\bar{x}(t))+\alpha V_{i}(\bar{x}(t)) \leqslant \varsigma^{\mathrm{T}}(t) \Lambda_{i} \varsigma(t)+\frac{1}{2} \gamma^{2} g_{i}^{\mathrm{T}}(t) g_{i}(t) \leqslant \\
& \varsigma^{\mathrm{T}}(t)\left[\begin{array}{ccc}
-\bar{Q} & 0 & 0 \\
0 & 0 & 0 \\
0 & 0 & 0
\end{array}\right] \varsigma(t)+\frac{1}{2} \gamma^{2} g_{i}^{\mathrm{T}}(t) g_{i}(t)= \\
& -\hat{e}_{r}^{\mathrm{T}}(t) \hat{e}_{r}(t)+\frac{1}{2} \gamma^{2} g_{i}^{\mathrm{T}}(t) g_{i}(t) .
\end{aligned}
$$

Letting $Q=\frac{1}{2} I \in \mathbf{R}^{n \times n}$, by Lemma 1, we can get

$$
\begin{aligned}
-\hat{e}_{r}^{\mathrm{T}}(t) \hat{e}_{r}(t) & =-\left[e(t)-e_{r}(t)\right]^{\mathrm{T}}\left[e(t)-e_{r}(t)\right]= \\
- & e_{r}^{\mathrm{T}}(t) e_{r}(t)-e^{\mathrm{T}}(t) e(t)+2 e_{r}^{\mathrm{T}}(t) e(t) \leqslant \\
- & e_{r}^{\mathrm{T}}(t) e_{r}(t)-e^{\mathrm{T}}(t) e(t)+e_{r}^{\mathrm{T}}(t) Q e_{r}(t)+ \\
e^{\mathrm{T}}(t) Q^{-1} e(t) & =-\frac{1}{2} e_{r}^{\mathrm{T}}(t) e_{r}(t)+\|e(t)\|^{2} .
\end{aligned}
$$

Similar to the reasoning in the above proof, let $e_{t}\left(t_{0}, \psi_{i_{0}}, \omega\right)$ denote the solution of system (8) with the initial condition $\left(t_{0}, \psi_{i_{0}}\right), \psi_{i_{0}}=e\left(t_{0}\right)=0, T_{1}\left(t, t_{0}\right)$ denotes a continuous piecewise linear operator. As the nominal system of (8) is exponentially stable, by Lemma 3, there exist $\kappa_{2}>0, \eta_{2}>0$ such that

$$
\begin{aligned}
& e_{t}\left(t_{0}, \psi_{i_{0}}, \omega\right)=T_{1}\left(t, t_{0}\right) \psi_{i_{0}}+\int_{t_{0}}^{t} T_{1}(t, s) X_{0} \omega(s) \mathrm{d} s \\
& \left\|T_{1}\left(t, t_{0}\right)\right\| \leqslant \kappa_{2} \mathrm{e}^{-\eta_{2}\left(t-t_{0}\right)} \\
& \left\|T_{1}(t, s) X_{0}\right\| \leqslant \kappa_{2} \mathrm{e}^{-\eta_{2}(t-s)}, \quad t \geqslant s \geqslant t_{0}
\end{aligned}
$$

According to Cauchy-Schwartz Inequality, one can get from (49):

$$
\begin{gathered}
\|e(t)\|^{2} \leqslant \int_{t_{0}}^{t} \kappa_{2}^{2} \mathrm{e}^{-\eta_{2}(t-s)} \mathrm{d} s \int_{t_{0}}^{t} \mathrm{e}^{-\eta_{2}(t-s)}\|\omega(s)\|^{2} \mathrm{~d} s \leqslant \\
\frac{\kappa_{2}^{2}}{\eta_{2}} \int_{t_{0}}^{t} \mathrm{e}^{-\eta_{2}(t-s)}\|\omega(s)\|^{2} \mathrm{~d} s .
\end{gathered}
$$

Let $\lambda_{2}=\max _{i \in M}\left\{\sigma_{\max }\left(L_{i} C_{i}\right)\right\}$, it has

$$
\begin{aligned}
g_{i}^{\mathrm{T}}(t) g_{i}(t)= & e^{\mathrm{T}}(t) C_{i}^{\mathrm{T}} L_{i}^{\mathrm{T}} L_{i} C_{i} e(t)+r^{\mathrm{T}}(t) r(t) \leqslant \\
& \lambda_{2}^{2}\|e(t)\|^{2}+r^{\mathrm{T}}(t) r(t) .
\end{aligned}
$$

Combining (47), (48), (50) and (51), it holds that

$$
\begin{aligned}
& \dot{V}_{i}(\bar{x}(t))+\alpha V_{i}(\bar{x}(t)) \leqslant-\frac{1}{2} e_{r}^{\mathrm{T}}(t) e_{r}(t)+\left(1+\frac{1}{2} \gamma^{2} \lambda_{2}^{2}\right) \\
& \frac{\kappa_{2}^{2}}{\eta_{2}} \int_{t_{0}}^{t} \mathrm{e}^{-\eta_{2}(t-s)}\|\omega(s)\|^{2} \mathrm{~d} s+\frac{1}{2} \gamma^{2} r^{\mathrm{T}}(t) r(t), \\
& t \in\left[t_{k}, t_{k+1}\right) \text {. } \\
& V_{i_{k}}(\bar{x}(t))=V_{i_{k}}\left(\bar{x}\left(t_{k}\right)\right)+\int_{t_{k}}^{t} \dot{V}_{i_{k}}(\bar{x}(s)) \mathrm{d} s \leqslant \\
& V_{i_{k}}\left(\bar{x}\left(t_{k}\right)\right)-\alpha \int_{t_{k}}^{t} V_{i_{k}}(\bar{x}(s)) \mathrm{d} s+\int_{t_{k}}^{t} \Gamma(s) \mathrm{d} s \leqslant \\
& \mu_{2} V_{i_{k-1}}\left(\bar{x}\left(t_{k-1}\right)\right)-\mu_{2} \alpha \int_{t_{k-1}}^{t_{k}} V_{i_{k-1}}(\bar{x}(s)) \mathrm{d} s+ \\
& \mu_{2} \int_{t_{k-1}}^{t_{k}} \Gamma(s) \mathrm{d} s-\alpha \int_{t_{k}}^{t} V_{i_{k}}(\bar{x}(s)) \mathrm{d} s+\int_{t_{k}}^{t} \Gamma(s) \mathrm{d} s \leqslant \cdots \leqslant \\
& \mu_{2}^{k} V_{i_{0}}\left(\bar{x}\left(t_{0}\right)-\mu_{2}^{k} \alpha \int_{t_{0}}^{t_{1}} V_{i_{0}}(\bar{x}(s)) \mathrm{d} s+\right. \\
& \mu_{2}^{k} \int_{t_{0}}^{t_{1}} \Gamma(s) \mathrm{d} s-\cdots-\alpha \int_{t_{k}}^{t} V_{i_{k}}(\bar{x}(s)) \mathrm{d} s+\int_{t_{k}}^{t} \Gamma(s) \mathrm{d} s \leqslant \\
& \mu_{2}^{N_{\sigma}\left(t, t_{0}\right)} V_{i_{0}}\left(\bar{x}\left(t_{0}\right)-\alpha \int_{t_{0}}^{t} V_{i_{k}}(\bar{x}(s)) \mathrm{d} s+\right. \\
& \int_{t_{0}}^{t} \mu_{2}^{N_{\sigma}(t, s)} \Gamma(s) \mathrm{d} s .
\end{aligned}
$$

Under zero initial condition, $V\left(\bar{x}\left(t_{0}\right)\right)=0$, and $V_{i_{k}}(\bar{x}(t)) \geqslant$ 0 , we can get

$$
\int_{t_{0}}^{t} \mu_{2}^{N_{\sigma}(t, s)} \Gamma(s) \mathrm{d} s \geqslant 0 .
$$

When $t \rightarrow \infty$, pre- and post-multiplying (54) by $\mathrm{e}^{-N_{\sigma}\left(t, t_{0}\right) \ln \mu_{2}}$ and letting $\frac{2 \kappa_{2}^{2}}{\eta_{2}^{2}-\kappa_{2}^{2} \lambda_{2}^{2}} \leqslant \gamma^{2}$, it can be concluded that

$$
\begin{aligned}
& \frac{1}{2} \int_{t_{0}}^{\infty} e_{r}^{\mathrm{T}}(s) e_{r}(s) \mathrm{e}^{-N_{\sigma}\left(s, t_{0}\right) \ln \mu_{2}} \mathrm{~d} s \leqslant \\
& \left(1+\frac{1}{2} \gamma^{2} \lambda_{2}^{2}\right) \frac{\kappa_{2}^{2}}{\eta_{2}} \int_{t_{0}}^{\infty} \int_{t_{0}}^{s} \mathrm{e}^{-\eta_{2}(s-\theta)}\|\omega(\theta)\|^{2} \mathrm{~d} \theta \mathrm{d} s+ \\
& \frac{1}{2} \gamma^{2} \int_{t_{0}}^{\infty} r^{\mathrm{T}}(s) r(s) \mathrm{d} s= \\
& \left(1+\frac{1}{2} \gamma^{2} \lambda_{2}^{2}\right) \frac{\kappa_{2}^{2}}{\eta_{2}^{2}} \int_{t_{0}}^{\infty}\|\omega(s)\|^{2} \mathrm{~d} s+ \\
& \frac{1}{2} \gamma^{2} \int_{t_{0}}^{\infty} r^{\mathrm{T}}(s) r(s) \mathrm{d} s \leqslant \frac{1}{2} \gamma^{2} \int_{t_{0}}^{\infty} \bar{\omega}^{\mathrm{T}}(s) \bar{\omega}(s) \mathrm{d} s
\end{aligned}
$$

By Definition 3 and (33), $N_{\sigma}\left(s, t_{0}\right) \ln \mu_{2} \leqslant \frac{s-t_{0}}{\tau_{a_{2}}} \ln \mu_{2} \leqslant$ $\alpha\left(s-t_{0}\right)$, it follows from (55) that

$$
\frac{1}{2} \int_{t_{0}}^{\infty} \mathrm{e}^{-\alpha\left(t-t_{0}\right)} e_{r}^{\mathrm{T}}(t) e_{r}(t) \mathrm{d} t \leqslant \frac{1}{2} \gamma^{2} \int_{t_{0}}^{\infty} \bar{\omega}^{\mathrm{T}}(t) \bar{\omega}(t) \mathrm{d} t .
$$

Remark 1. The average dwell-time $\tau_{a 1}$ is designed to guarantee that system (17) is exponentially stable, and $\tau_{a 2}$ 
can guarantee system (36) is exponentially stable. The average dwell-time $\tau_{a}$ of the whole system is chosen as the maximum value between $\tau_{a 1}$ and $\tau_{a 2}$, which can guarantee that system (8) and (9) are exponentially stable with $H_{\infty}$ performance index. In [21], a condition is required to hold that (8) has the common Lyapunov function and the designed switching law relies on the states $\bar{x}(t), \dot{\bar{x}}(t)$ and $\dot{\bar{x}}(s)$ which contains the derivative term. The proposed method just needs that (8) satisfies the average dwell-time (19) lowering the conservatism, which is one of the main contributions of this paper. The asymptotic stability of system (10) is obtained in [21], while the proposed method can guarantee that system (10) is exponentially stable with $g_{i}(t) \neq 0$.

\section{$3.3 \quad H_{\infty}$ controller design}

First, let

$$
\begin{aligned}
& \Omega_{11 i}=\left[\begin{array}{cc}
\psi_{11 i} & B_{i} \bar{F}_{i} \\
* & \psi_{22 i}
\end{array}\right], \Omega_{12 i}=\left[\begin{array}{cc}
D_{i} S_{1 i} & 0 \\
0 & 0
\end{array}\right] \\
& \Omega_{14 i}=\left[\begin{array}{cc}
\psi_{17 i} & 0 \\
\bar{F}_{i}^{\mathrm{T}} B_{i}^{\mathrm{T}} & X_{2 i} A_{r}^{\mathrm{T}}
\end{array}\right], \Omega_{15 i}=\left[\begin{array}{c}
X_{1 i} \\
-X_{2 i}
\end{array}\right]
\end{aligned}
$$

where $\psi_{11 i}=X_{1 i}\left(A_{i}+D_{i}\right)^{\mathrm{T}}+\left(A_{i}+D_{i}\right) X_{1 i}+\alpha X_{1 i}+B_{i} \bar{K}_{i}+$ $\bar{K}_{i}^{\mathrm{T}} B_{i}^{\mathrm{T}}, \psi_{22 i}=A_{r} X_{2 i}+X_{2 i} A_{r}^{\mathrm{T}}+\alpha X_{2 i}, \psi_{17 i}=X_{1 i}\left(A_{i}+\right.$ $\left.D_{i}\right)^{\mathrm{T}}+\bar{K}_{i}^{\mathrm{T}} B_{i}^{\mathrm{T}}$, and $\Omega_{24 i}=\Omega_{12 i}^{\mathrm{T}}$.

Theorem 3. Consider the augmented system (10), and suppose that Assumptions 1 and 2 and (18)-(20) hold. For the given scalars $\tau>0, \alpha>0, \gamma>0$, there exist matrices $X_{1 i}>0, X_{2 i}>0, S_{1 i}>0, S_{2 i}>0, \bar{K}_{i}, \bar{F}_{i}, \forall i \in M$, such that the following LMIs hold

$$
\Omega_{i}=\left[\begin{array}{ccccc}
\Omega_{11 i} & \Omega_{12 i} & I & \tau \Omega_{14 i} & \Omega_{15 i} \\
* & -\mathrm{e}^{-\alpha \tau} \bar{S}_{i} & 0 & \tau \Omega_{24 i} & 0 \\
* & * & -\frac{1}{2} \gamma^{2} I & \tau I & 0 \\
* & * & * & -\bar{S}_{i} & 0 \\
* & * & * & * & -I
\end{array}\right]<0
$$

and average dwell-time satisfies (33), $\mu_{2} \geqslant 1$ satisfies

$$
\begin{aligned}
& X_{1 i} \leqslant \mu_{2} X_{1 j}, X_{2 i} \leqslant \mu_{2} X_{2 j}, \\
& S_{1 i} \leqslant \mu_{2} S_{1 j}, S_{2 i} \leqslant \mu_{2} S_{2 j}, \quad \forall i, j \in M, i \neq j .
\end{aligned}
$$

Then, the system (10) is exponentially stable, and (1) has the $H_{\infty}$ model reference tracking performance. The designed controller gain matrices are given by $K_{i}=$ $\bar{K}_{i} X_{1 i}^{-1}, F_{i}=\bar{F}_{i} X_{2 i}^{-1}$.

Proof. Choose the form of positive definite matrices as $P_{i}=\left[\begin{array}{cc}P_{1 i} & 0 \\ * & P_{2 i}\end{array}\right], S_{i}=\left[\begin{array}{cc}\hat{S}_{1 i} & 0 \\ * & \hat{S}_{2 i}\end{array}\right]$. $\quad$ Preand post-multiplying (32) by $\operatorname{diag}\left\{P_{i}^{-1}, S_{i}^{-1}, I, I\right\}$, denote $X_{i}=P_{i}^{-1}=\left[\begin{array}{cc}X_{1 i} & 0 \\ * & X_{2 i}\end{array}\right], \bar{S}_{i}=S_{i}^{-1}=$ $\left[\begin{array}{cc}S_{1 i} & 0 \\ * & S_{2 i}\end{array}\right], \bar{K}_{i}=K_{i} X_{1 i}, \bar{F}_{i}=F_{i} X_{2 i}$, and $X_{i} \bar{Q} X_{i}=$ $\left.\begin{array}{c}X_{1 i} \\ -X_{2 i}\end{array}\right]\left[\begin{array}{ll}X_{1 i} & -X_{2 i}\end{array}\right]$. By using Schur complement for (32) in Theorem 2, we can easily get (56).

Remark 2. The work in [22] proposed an exponential stability criterion for a class of switched nonlinear systems with time-varying delay. However, the designed controller relies on the system state $x(t)$ and reference state $x_{r}(t)$. In this paper, we design an observer to deal with the unavailable state $x(t)$ and construct an output feedback controller to track the reference signal $x_{r}(t)$.

\section{Numerical example}

Consider system (1) and reference system (5) with

$$
\begin{aligned}
& A_{1}=\left[\begin{array}{ll}
3.5 & 1.5 \\
1.5 & 4.5
\end{array}\right], B_{1}=\left[\begin{array}{cc}
-0.6 & -0.2 \\
0.2 & -0.4
\end{array}\right] \\
& C_{1}=\left[\begin{array}{ll}
-0.2 & 0.8
\end{array}\right], D_{1}=\left[\begin{array}{cc}
0.25 & 0.15 \\
0.25 & -0.15
\end{array}\right] \\
& A_{2}=\left[\begin{array}{cc}
4.5 & 2 \\
-1.5 & 3
\end{array}\right], B_{2}=\left[\begin{array}{cc}
-0.5 & 0.1 \\
-0.1 & -0.25
\end{array}\right] \\
& C_{2}=\left[\begin{array}{cc}
1.0 & -0.7
\end{array}\right], D_{2}=\left[\begin{array}{cc}
-0.1 & 0.1 \\
0.2 & 0
\end{array}\right] \\
& A_{r}=\left[\begin{array}{cc}
-5 & 0.5 \\
-1.5 & -4.5
\end{array}\right], f_{1}(x(t))=\left[\begin{array}{c}
0.1 \cos \left(0.01 x_{1}\right) \\
0.1 \cos \left(0.01 x_{2}\right)
\end{array}\right] \\
& f_{2}(x(t))=\left[\begin{array}{c}
0.2 \cos \left(0.01 x_{1}\right) \\
0.2 \cos \left(0.01 x_{2}\right)
\end{array}\right] .
\end{aligned}
$$

We adopt the parameters below: $d(t)=0.16+0.14 \sin t, \tau=$ $0.3, \alpha=1, \gamma=0.5$, and the Lipschitz matrices are given by

$$
U_{1}=\left[\begin{array}{cc}
0.1 & 0 \\
0 & 0.1
\end{array}\right], \quad U_{2}=\left[\begin{array}{cc}
0.2 & 0 \\
0 & 0.2
\end{array}\right]
$$

First, let $\mu_{1}=7$, by Theorem 1 , we obtain

$$
\begin{aligned}
H_{1} & =\left[\begin{array}{cc}
0.6095 & -1.3686 \\
-1.3686 & 3.3900
\end{array}\right], L_{1}=\left[\begin{array}{c}
73.9087 \\
46.4478
\end{array}\right] \\
H_{2} & =\left[\begin{array}{cc}
1.0839 & -1.2598 \\
-1.2598 & 1.6531
\end{array}\right], L_{2}=\left[\begin{array}{c}
23.8485 \\
9.8203
\end{array}\right] \\
G_{1} & =\left[\begin{array}{cc}
0.3475 & -0.6217 \\
-0.6217 & 1.2153
\end{array}\right], G_{2}=\left[\begin{array}{cc}
0.4400 & -0.6056 \\
-0.6056 & 0.9653
\end{array}\right]
\end{aligned}
$$

and the average dwell-time satisfies $\tau_{a_{1}}>1.9459 \mathrm{~s}$. However, by using the proposed method in [21], we cannot obtain the feasible solutions, so we cannot get the corresponding common Lyapunov function.

Then, considering the system (9), and using Theorem 3, we can get the common Lyapunov function and a set of solutions

$$
\begin{aligned}
& X_{1 i}=\left[\begin{array}{cc}
2.0301 & 0 \\
0 & 1.9886
\end{array}\right], X_{2 i}=\left[\begin{array}{cc}
2.5081 & -0.0537 \\
-0.0537 & 2.7959
\end{array}\right] \\
& S_{1 i}=\left[\begin{array}{cc}
21.0670 & -0.0569 \\
-0.0569 & 22.7170
\end{array}\right], S_{2 i}=\left[\begin{array}{cc}
24.4292 & -0.0840 \\
-0.0840 & 24.3988
\end{array}\right] \\
& K_{1}=\left[\begin{array}{cc}
14.3934 & -5.6477 \\
12.1647 & 26.0282
\end{array}\right], K_{2}=\left[\begin{array}{cc}
20.2412 & 11.0896 \\
-13.5763 & 35.8298
\end{array}\right] \\
& F_{1}=\left[\begin{array}{cc}
-2.6262 & 1.3188 \\
-1.1708 & -3.7332
\end{array}\right], F_{2}=\left[\begin{array}{cc}
-3.5098 & -1.3707 \\
1.3485 & -6.7497
\end{array}\right]
\end{aligned}
$$

where $i=1,2$, thus, we can get the average dwell-time $\tau_{a_{2}}>0$. 
According to the switching law (33), we choose the average dwell-time $\tau_{a}=2 \mathrm{~s}$. Let $w(t)=\left[\begin{array}{l}w_{1}(t) \\ w_{2}(t)\end{array}\right], r(t)=$ $\left[\begin{array}{l}r_{1}(t) \\ r_{2}(t)\end{array}\right], \quad \omega_{i}(t)=r_{i}(t)=\left\{\begin{array}{l}2 \frac{\sin t}{t}, \quad 5 \mathrm{~s} \leqslant t \leqslant 20 \mathrm{~s} \\ 0, \quad \text { others }\end{array}, \quad i=1,2\right.$, and choose the initial conditions $\varphi(t)=0, t \in$ $[-0.3,0), x(0)=[0.4,-0.3]^{\mathrm{T}}, \hat{x}(0)=[0.3,-0.2]^{\mathrm{T}}, x_{r}(t)=$ $[0.6,-0.5]^{\mathrm{T}}$. Then, the simulation results are shown in Figs. 1-4.

It can be seen that the state error is exponentially stable after $2 \mathrm{~s}$ when $\bar{\omega}(t)=0$, and the real state tracks the reference state perfectly. When the system is subjected to the bounded exogenous disturbance, we can see that it has $H_{\infty}$ tracking performance under the designed controller and switching law.

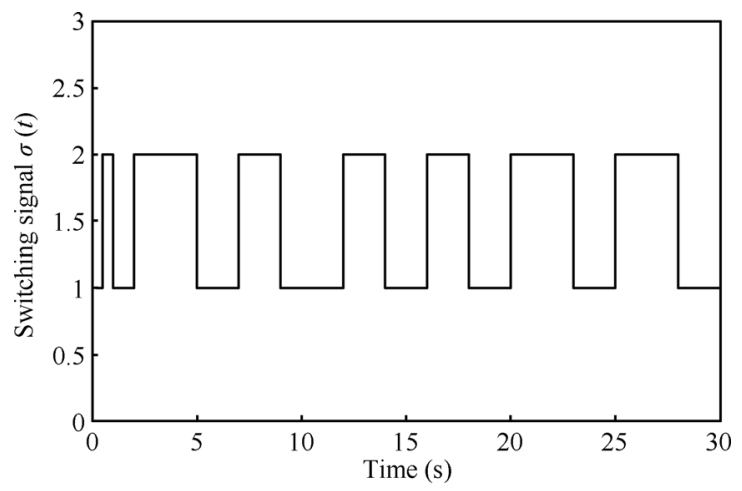

Fig. 1 The designed switching signal $\sigma(t)$

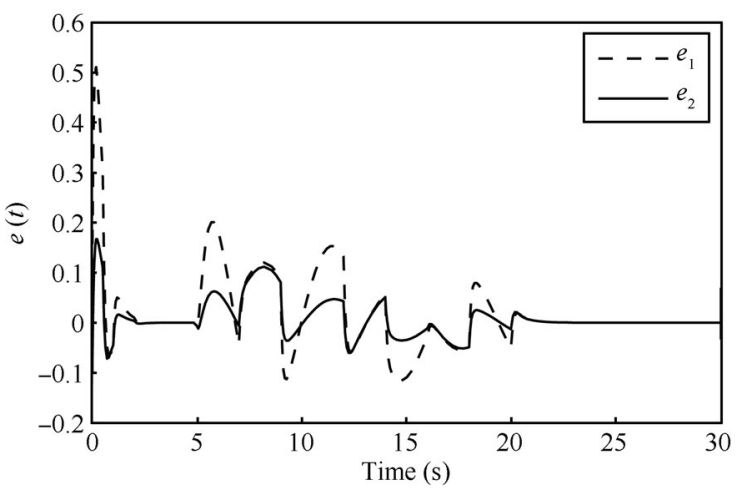

Fig. 2 State error $e(t)$

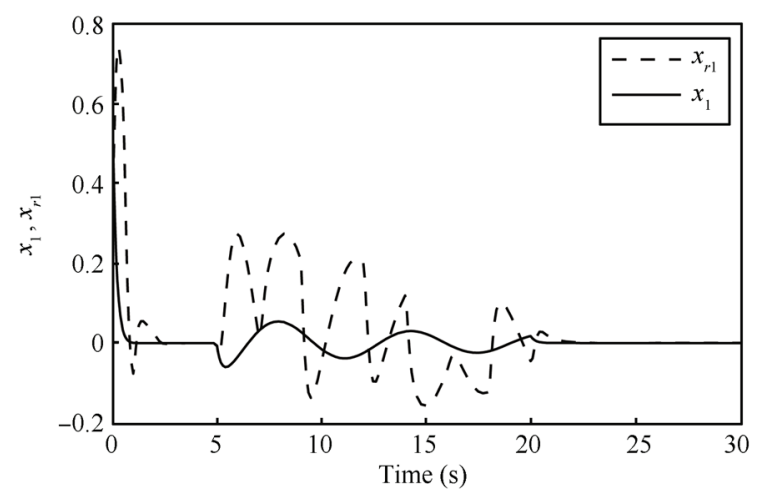

Fig. 3 The state $x_{1}$ and the reference state $x_{r 1}$

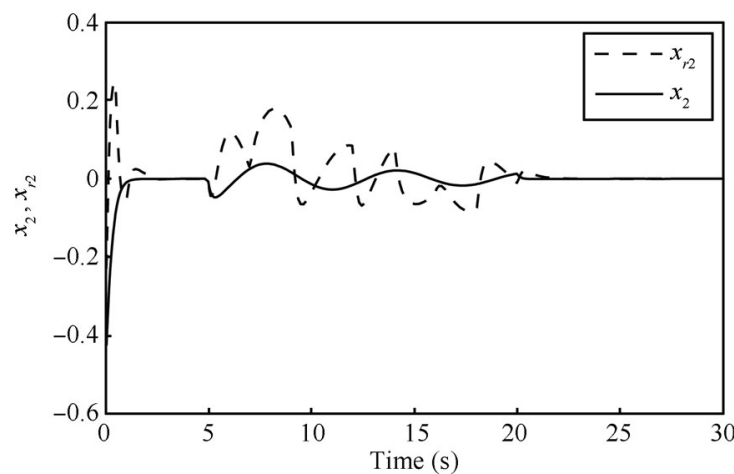

Fig. 4 The state $x_{2}$ and the reference state $x_{r 2}$

\section{Conclusions}

In this paper, output feedback tracking control for switched nonlinear system with time-varying delay has been studied. A new switching law is designed and exponential stability criterion for the system is derived. Average dwelltime and piecewise Lyapunov function have been used to obtain the stability of the augmented system and $H_{\infty}$ tracking performance when the system has the bounded exogenous disturbance. Besides, the designed observer and controller can be obtained by solving a set of LMIs, and the proposed method does not need that a common Lyapunov function exists for the switched nonlinear systems. A numerical example is given to show the effectiveness of the proposed method. If the nonlinear function $f_{\sigma}(\cdot)$ is unknown, how to deal with this problem will be our further study issue.

\section{References}

[1] J. P. Hespanha, A. S. Morse. Stability of switched systems with average dwell-time. In Proceedings of the 38th Conference on Decision \& Control, IEEE, Phoenix, Arizona, USA, pp. 2655-2660, 1999.

[2] G. S. Zhai, B. Hu, K. Yasuda, A. N. Michel. Stability analysis of switched systems with stable and unstable subsystems: An average dwell time approach. In Proceedings of the American Control Conference, IEEE, Chicago, USA, pp. 200-204, 2000.

[3] H. Lin, P. J. Antsaklis. Stability and stabilizability of switched linear systems: A survey of recent results. IEEE Transactions on Automatic Control, vol.54, no. 2, pp. 308$322,2009$.

[4] W. M. Xiang, J. Xiao, M. N. Iqbal. Robust observer design for nonlinear uncertain switched systems under asynchronous switching. Nonlinear Analysis: Hybrid Systems, vol. 6, no. 1, pp. 754-773, 2012.

[5] D. W. Ding, G. H. Yang. $H_{\infty}$ static output feedback control for discrete-time switched linear systems with average dwell time. IET Control Theory and Applications, vol. 4, no. 3, pp. 381-390, 2010.

[6] J. Y. Zhai, W. T. Zha. Global adaptive output feedback control for a class of nonlinear time-delay systems. ISA Transactions, vol. 53, no. 1, pp. 2-9, 2014.

[7] W. T. Zha, J. Y. Zhai, S. M. Fei. Output feedback control for a class of stochastic high-order nonlinear systems with time-varying delays. International Journal of Robust and Nonlinear Control, vol. 24, no. 16, pp. 2243-2260, 2014.

[8] T. Wang, M. X. Xue, C. Zhang, S. M. Fei. Improved stability criteria on discrete-time systems with time-varying and distributed delays. International Journal of Automation and Computing, vol. 10, no. 3, pp. 260-266, 2013. 
[9] Y. Y. Cao, Y. X. Sun, C. W. Cheng. Delay-dependent robust stabilization of uncertian systems with multiple state delays. IEEE Transactions on Automatic Control, vol. 43, no. 11, pp. 1608-1612, 1998.

[10] X. M. Sun, J. Zhao, D. J. Hill. Stability and $L_{2}$-gain analysis for switched delay systems: A delay-dependent method. Automatica, vol. 42, no. 10, pp. 1769-1774, 2006.

[11] J. Liu, X. Z. Liu, W. C. Xie. Delay-dependent robust control for uncertain switched systems with time-delay. Nonlinear Analysis: Hybrid Systems, vol. 2, no. 1, pp. 81-95, 2008.

[12] L. V. Hien, Q. P. Ha, V. N. Phat. Stability and stabilization of switched linear dynamic systems with time delay and uncertainties. Applied Mathematics and Computation, vol. 201, no. 1, pp. 223-231, 2009.

[13] X. M. Sun, W. Wang, G. P. Liu, J. Zhao. Stability analysis for linear switched systems with time-varying delay. IEEE Transactions on Systems, Man, and Cybernetics, Part B: Cybernetics, vol. 38, no. 2, pp. 528-533, 2008.

[14] H. L. Xu, X. Z. Liu, K. L. Teo. A LMI approach to stability analysis and synthesis of impulsive switched systems with time delays. Nonlinear Analysis: Hybrid Systems, vol. 2, no. 1 , pp. 38-50, 2008.

[15] Y. G. Sun, L. Wang, G. M. Xie. Delay-dependent robust stability and stabilization for discrete-time switched systems with mode-dependent time-varying delays. Applied Mathematics and Computation, vol. 180, no. 2, pp. 428-435, 2006.

[16] X. H. Liu. Stabilization of switched linear systems with mode-dependent time-varying delays. Applied Mathematics and Computation, vol. 216, no. 9, pp. 2581-2586, 2010.

[17] S. Cong, S. M. Fei, T. Li. Exponential stability of switched systems with delay: A multiple Lyapunov function approach. Acta Automatica Sinica, vol. 33, no. 9, pp. 985-988, 2007. (in Chinese)

[18] S. L. Liu, Z. R. Xiang, Q. W. Chen. Stability and stabilization of a class of switched nonlinear systems with time-varying delay. Applied Mathematics and Computation, vol. 218, no. 23, pp. 11534-11546, 2012.

[19] Q. K. Li, J. Zhao, G. M. Dimirovski, X. J. Liu. Tracking control for switched linear systems with time-delay: A state-dependent switching method. Asian Journal of Control, vol. 11, no. 5, pp. 517-526, 2009.

[20] Q. K. Li, J. Zhao, G. M. Dimirovski. Robust tracking control for switched linear systems with time-varying delays. IET Control Theory and Applications, vol. 2, no. 6, pp. 449457, 2008.

[21] Q. K. Li, J. Zhao, X. J. Liu, G. M. Dimirovski. Observerbased tracking control for switched linear systems with time-varying delay. International Journal of Robust and Nonlinear Control, vol. 21, no. 3, pp. 309-327, 2011.

[22] Z. R. Xiang, S. L. Liu, Q. W. Chen. Tracking control for a class of switched non-linear systems with time-varying delay. Transactions of the Institute of Measurement and Control, vol. 35, no. 3, pp. 398-406, 2013.

[23] J. Lian, Y. L. Ge. Robust $H_{\infty}$ output tracking control for switched systems under asynchronous switching. Nonlinear Analysis: Hybrid Systems, vol. 8, pp. 57-68, 2013.

[24] S. L. Liu, Z. R. Xiang. Exponential $L_{1}$ output tracking control for positive switched linear systems with time-varying delays. Nonlinear Analysis: Hybrid Systems, vol.11, no. 1, pp. 118-128, 2014.

[25] S. R. Liu, Z. R. Xiang. Exponential $H_{\infty}$ output tracking control for switched neutral system with time-varying delay and nonlinear perturbations. Circuits, Systems, and Signal Processing, vol. 32, no. 1, pp. 103-121, 2013.

[26] M. Rehan, K. S. Hong, S. S. Ge. Stabilization and tracking control for a class of nonlinear systems. Nonlinear Analysis: Real World Applications, vol. 12, no. 3, pp. 1786-1796, 2011.

[27] J. K. Hale. Theory of Functional Differential Equations, Berlin, Germany: Springer Press, pp. 141-164, 1977.

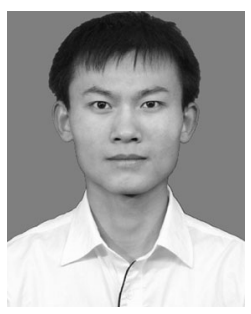

Bin Wang received his B.Sc. degree from Electrical and Electronic Engineering College, Chang Chun University of Technology, China in 2011. Now, he is a master student in the School of Automation at Southeast University, China.

His research interests include robust control and integration of switched systems.

E-mail: ccutbw@163.com

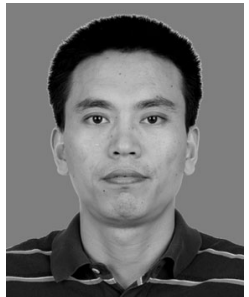

ing author)

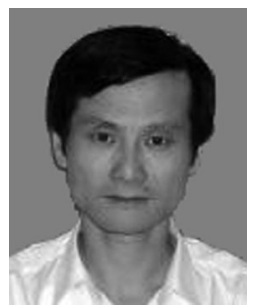

and complex systems.

E-mail: smfei@seu.edu.cn trol.

Jun-Yong Zhai received his $\mathrm{Ph}$. D. degree in automatic control from Southeast University, China in 2006. He is currently a professor and doctoral advisor in the School of Automation, Southeast University.

His research interests include nonlinear systems control, stochastic time-delay systems, and multiple models switching con-

E-mail: jyzhai@seu.edu.cn (Correspond-

Shu-Min Fei received his Ph. D. degree from Beijing University of Aeronautics and Astronautics, China in 1995. From 1995 to 1997, he was a postdoctoral research fellow at Southeast University. Presently, he is a professor and doctoral advisor at Southeast University. He has published more than 70 journal papers.

His research interests include nonlinear systems, stability theory of delayed system, 